\title{
REPRESENTATIONS OF ALTERNATIVE ALGEBRAS
}

\author{
BY \\ R. D. SCHAFER( ${ }^{(1)}$
}

In this paper we apply to alternative algebras a definition of representation given by $\mathrm{S}$. Eilenberg for nonassociative algebras satisfying multilinear identities. The corresponding alternative module generalizes the notion of a two-sided $\mathfrak{A}$-module as used in the study of associative algebras.

Our chief object is to use the representation theory to obtain the generalization to alternative algebras of the theorem of A. Malcev on the strict conjugacy of semisimple components in Wedderburn decompositions. Since every alternative algebra gives rise to a (special) Jordan algebra, and every representation yields (Jordan) representations of this algebra, we can use recent results of $\mathrm{N}$. Jacobson on representations of Jordan algebras. Doing this restricts our principal theorems to algebras of characteristic 0 .

Following certain preliminaries concerning derivations and associators, we prove the complete reducibility of representations of semisimple alternative algebras. We next prove the first Whitehead lemma for alternative algebras, generalizing G. Hochschild's result for associative algebras. This is sufficient to prove the Malcev theorem in case the square of the radical is $\{0\}$. For all other types of algebras for which this theorem is known (Lie, associative, and Jordan), an inductive argument then suffices to complete the proof for an arbitrary radical. In the case of alternative algebras, however, without a stronger form of the Whitehead lemma a certain associativity condition would invalidate the inductive argument. Using the complete reducibility, we prove that this stronger form holds, and employ it in the proof of the Malcev theorem.

In the concluding section we prove a generalization of a theorem due to Hochschild which, although independent of the representation theory, is related to our other results: an alternative algebra (of characteristic 0 ) is semisimple if and only if its derivation algebra is semisimple or $\{0\}$.

We are indebted to Professor Jacobson for allowing us to see his paper, General representation theory of Jordan algebras, in manuscript form, and also for giving us valuable advice in connection with the proof of Theorem 2 .

1. Representations and semidirect sums. A (nonassociative) algebra $\mathfrak{A}$ over a field $F$ is called alternative in case

$$
x(y y)=(x y) y, \quad(y y) x=y(y x)
$$

for all $x, y$ in $\mathfrak{A}$. Clearly every associative algebra is alternative. Linearization

Presented to the Society, April 28, 1951; received by the editors March 14, 1951.

(1) This research was supported in part by a contract with the Office of Naval Research. 
of the identities (1) yields the fact that the associator

$$
(x, y, z)=(x y) z-x(y z)
$$

"alternates": it changes sign under an odd permutation of the letters $x, y, z$, but remains unchanged under an even permutation. The most economical statement of this fact is

$$
(x, y, z)=-(y, x, z)=(z, x, y)
$$

for all $x, y, z$ in $\mathfrak{A}$. If the characteristic of $F$ is not two, then the identities (3) define an alternative algebra.

Let $\mathfrak{B}$ be a vector space over $F$. Following Eilenberg $[3, \S 2]\left({ }^{2}\right)$, we define a representation of $\mathfrak{A}$ as a pair of linear mappings $x \rightarrow S_{x}, x \rightarrow T_{x}$ of $\mathfrak{A}$ into the algebra of all linear transformations on $\mathfrak{B}$, satisfying

$$
\left[T_{x}, S_{z}\right]=S_{x z}-S_{x} S_{z}=T_{z x}-T_{x} T_{z}=\left[S_{x}, T_{z}\right]
$$

for all $x, z$ in $\mathfrak{A}$, where $[X, Y]$ denotes the commutator $X Y-Y X$. We write $(S, T)$ for the representation. The representation space $\mathfrak{B}$ in which $(S, T)$ acts is made into an alternative module by defining

$$
v x=v S_{x}, \quad x v=v T_{x}
$$

for $v$ in $\mathfrak{B}, x$ in $\mathfrak{A}$. Assumption (4) becomes

$$
(x, v, z)=-(v, x, z)=(z, x, v)=-(z, v, x)
$$

for all $x, z$ in $\mathfrak{A}, v$ in $\mathfrak{B}$, where the associator is defined as in (2) except that one argument is in $\mathfrak{B}$. The identities (5) are equivalent to the assumption that every associator with one argument in $\mathfrak{B}$ and two in $\mathfrak{A}$ "alternates." It cannot be reduced to the equality of three terms (as in (3)) because the arguments do not enter symmetrically. The concept of an alternative module is a generalization of that of a two-sided $\mathfrak{A}$-module $\left(^{3}\right)$ for associative algebras $\mathfrak{A}$. It should be noted that, even if a particular alternative algebra $\mathfrak{A}$ is associative, an alternative module for $\mathfrak{A}$ need not be a two-sided $\mathfrak{A}$-module; in the latter case all of the associators (5) would vanish $\left({ }^{4}\right)$.

Every alternative algebra $\mathfrak{A}$ over $F$ has a representation; namely, the regular representation $(R, L)$ where $R_{x}$ and $L_{x}$ are the right and left multiplications defined respectively by $y \rightarrow y x$ and $y \rightarrow x y$ for all $y$ in $\mathfrak{A}$. In this case $\mathfrak{A}$ itself is the alternative module. We have the identities

$$
\begin{aligned}
{\left[L_{x}, R_{z}\right] } & =R_{x z}-R_{x} R_{z}=L_{z x}-L_{x} L_{z} \\
& =\left[R_{x}, L_{z}\right]=R_{z} R_{x}-R_{z x}=L_{z} L_{x}-L_{x z} .
\end{aligned}
$$

(2) Numbers in brackets refer to the references cited at the end of the paper.

(3) See [5, Definition 2.1].

(4) For example, let $\mathfrak{A}$ be a quaternion subalgebra of a Cayley algebra $\mathfrak{C}$. Then $\mathfrak{S}$ is generated by $\mathfrak{A}$ and an element $z$ such that $(x, y, z) \neq 0$ for some $x, y$ in $\mathfrak{A}$. Hence $\mathfrak{C}$ is an alternative module for $\mathfrak{A}$, but not a two-sided $\mathfrak{A}$-module. 
More generally, if $\mathfrak{A}$ is any subalgebra of an alternative algebra $\mathfrak{B}$ over $F$ and if $\mathfrak{B}$ is an ideal of $\mathfrak{B}$, then the regular representation of $\mathfrak{B}$ induces a representation of $\mathfrak{A}$ acting in $\mathfrak{B}$.

For any representation $(S, T)$ of $\mathfrak{A}$ with corresponding alternative module $\mathfrak{B}$, we define an algebra $\mathfrak{B}=\mathfrak{A}+\mathfrak{B}$, called the semidirect sum of $\mathfrak{A}$ and $\mathfrak{B}$, as follows. Let $\mathfrak{B}$ be the direct sum of the vector spaces $\mathfrak{A}$ and $\mathfrak{B}$ over $F$, and define multiplication in $\mathfrak{B}$ by

$$
(x+v)\left(z+v^{\prime}\right)=x z+\left(v z+x v^{\prime}\right)=x z+\left(v S_{z}+v^{\prime} T_{x}\right)
$$

for $x, z$ in $\mathfrak{A}, v, v^{\prime}$ in $\mathfrak{B}$. Then it is readily verified that, since $\mathfrak{B}$ is an alternative module, $\mathfrak{B}$ is an alternative algebra. Moreover, $\mathfrak{B}$ is an ideal of $\mathfrak{B}, \mathfrak{B}^{2}=\{0\}$, and the representation $(S, T)$ of $\mathfrak{A}$ in $\mathfrak{B}$ is induced by the regular representation of $\mathfrak{B}$.

Let $(S, T)$ be a representation of $\mathfrak{A}$ acting in $\mathfrak{B}$. A subspace $\mathfrak{B}$ of $\mathfrak{B}$ is called invariant in case $\mathfrak{B} S_{x} \leqq \mathfrak{B}$ and $\mathfrak{B} T_{x} \leqq \mathfrak{B}$ for every $x$ in $\mathfrak{A}$; that is, $w x$ and $x w$ are in $\mathfrak{W}$ for every $w$ in $\mathfrak{B}$ and $x$ in $\mathfrak{A}$. We call $(S, T)$ an irreducible representation of $\mathfrak{A}$ in case the only subspaces of $\mathfrak{B}$ which are invariant are $\{0\}$ and $\mathfrak{B}$. Equivalently, the invariant subspaces of $\mathfrak{B}$ are those ideals of the semidirect sum $\mathfrak{B}=\mathfrak{A}+\mathfrak{B}$ which are contained in $\mathfrak{B}$, and a representation of $\mathfrak{A}$ is irreducible if and only if $\mathfrak{B}$ does not contain properly any nonzero ideal of $\mathfrak{B}$.

Let $\mathfrak{A}$ be any alternative algebra over $F$. A new algebra $\mathfrak{A}^{+}$may be defined by introducing a new multiplication

$$
x \cdot z=x z+z x
$$

in $\mathfrak{A}$. Then $\mathfrak{A}^{+}$is a special Jordan algebra (that is, is isomorphic to a Jordan algebra of matrices) $\left.{ }^{5}\right)$. For, if $\mathfrak{A}$ does not have a unity element, it is necessary only to adjoin one to $\mathfrak{A}$ to obtain $\mathfrak{A} \leqq \mathfrak{A}_{1}$, an alternative algebra with unity element. For $x$ in $\mathfrak{A} \leqq \mathfrak{A}_{1}$, consider the one-to-one linear mapping $x \rightarrow R_{x}$ where $R_{x}$ is the right multiplication for $\mathfrak{A}_{1}$. From (6) we have

$$
R_{x \cdot z}=R_{x z+z x}=R_{x} R_{z}+R_{z} R_{x},
$$

or $x \rightarrow R_{x}$ is an isomorphism between $\mathfrak{A}^{+}$and the special Jordan algebra of all the $R_{x}$.

Let $(S, T)$ be a representation of the alternative algebra $\mathfrak{A}$. Then $x \rightarrow S_{x}$ and $x \rightarrow T_{x}$ are special Jordan representations [7, §2] of $\mathfrak{A}^{+}$acting in $\mathfrak{B}$, since (4) (together with the equalities which follow by interchange of $x$ and $z$ ) implies $S_{x \cdot z}=S_{x z+z x}=S_{x} S_{z}+S_{z} S_{x}$ and $T_{x \cdot z}=T_{x z+z x}=T_{x} T_{z}+T_{z} T_{x}$. Also $x \rightarrow S_{x}^{+}$ where

$$
S_{x}^{+}=S_{x}+T_{x}
$$

is a (general) Jordan representation $S^{+}$of $\mathfrak{A}^{+}$. We may check this by direct

(5) See $[1,85]$. 
substitution of (7) and (4) in the defining identities for a Jordan representation, or more readily by the following observation: if $\mathfrak{B}$ is the semidirect sum $\mathfrak{B}=\mathfrak{U}+\mathfrak{B}$, then $\mathfrak{B}$ is alternative so that $\mathfrak{B}^{+}$is a (special) Jordan algebra. Now the elements of $\mathfrak{B}$ form a (Jordan) ideal of $\mathfrak{B}^{+}$. Therefore the (Jordan) regular representation of $\mathfrak{B}^{+}$induces a (Jordan) representation of the subalgebra $\mathfrak{A}^{+}$acting in $\mathfrak{B}$. This Jordan representation of $\mathfrak{A}^{+}$is exactly the mapping $S^{+}$. The representation space $\mathfrak{B}$, equipped with the new composition

$$
v \cdot x=x \cdot v=v S_{x}^{+}=v x+x v,
$$

is a Jordan module which we shall denote by $\mathfrak{B}^{+}$, and $\mathfrak{B}^{+}$is the (Jordan) semidirect sum $\mathfrak{B}^{+}=\mathfrak{U}^{+}+\mathfrak{B}^{+}$.

The radical $\mathfrak{N}$ of an alternative algebra $\mathfrak{A}$ is its maximal nilpotent ideal, and $\mathfrak{U}$ is called semisimple in case $\mathfrak{N}=\{0\}$. A. A. Albert has shown [1, p. 328] that, if $\mathfrak{A}$ is a (right) alternative algebra over $F$ of characteristic 0 , then the radical of the Jordan algebra $\mathfrak{A}^{+}$is exactly $\mathfrak{N}^{+}$. Hence, if $\mathfrak{N}$ is semisimple, then so is $\mathfrak{H}^{+}$.

2. Some lemmas on derivations and associators. A derivation of a nonassociative algebra $\mathfrak{A}$ over $F$ is a linear transformation $D$ on $\mathfrak{A}$ satisfying

$$
(x y) D=x(y D)+(x D) y
$$

for all $x, y$ in $\mathfrak{A}$. If $\mathfrak{A}$ is a subalgebra of a nonassociative algebra $\mathfrak{B}$, then a linear mapping $D$ of $\mathfrak{A}$ into $\mathfrak{B}$ satisfying $(8)$ is called a derivation of $\mathfrak{A}$ into $\mathfrak{B}$ $[5$, p. 688$]$. In $[10, \S 3]$ we determined the derivations of semisimple alternative algebras of characteristic $0\left(^{6}\right)$. We shall require some of the same formulas here.

It is readily verified that (6) implies

$$
\begin{aligned}
{\left[R_{x}, R_{z}\right] } & =R_{[x, z]}-2\left[L_{x}, R_{z}\right], \\
{\left[L_{x}, L_{z}\right] } & =-L_{[x, z]}-2\left[L_{x}, R_{z}\right], \\
{\left[L_{y},\left[L_{x}, L_{z}\right]\right] } & =L_{[y,[x, z]]-2(x, y, z),}
\end{aligned}
$$

and

$$
\left[R_{y},\left[R_{x}, R_{z}\right]\right]=R_{[y,[x, z]]-2(x, y, z)} .
$$

Also, if the characteristic of $F$ is not two, then

$$
\begin{aligned}
D_{x, z} & =\left[R_{x}, R_{z}\right]+\left[L_{x}, R_{z}\right]+\left[L_{x}, L_{z}\right] \\
& =R_{[x, z]}-L_{[x, z]}-3\left[L_{x}, R_{z}\right]
\end{aligned}
$$

is a derivation of $\mathfrak{A}$ for all $x, z$ in $\mathfrak{A}$, and hence

${ }^{(6)}$ We take this opportunity to remark that, whereas in [10] we proved that these derivations have the form (17), it is easy to see that they have the more special form (13). We have shown this for the Cayley components [10, Theorem 6]. For the associative components the conclusion follows from (25) and (12). See also Theorem 5 


$$
D=\sum D_{x_{i}, z_{i}}
$$

is a derivation of $\mathfrak{A}$.

If $\mathfrak{A}$ is any nonassociative algebra over $F$, then the subset $\mathcal{B}$ of $\mathfrak{A}$ consisting of all $g$ in $\mathfrak{A}$ satisfying

$$
(x, y, g)=(x, g, y)=(g, x, y)=0,
$$

for all $x, y$ in $\mathfrak{A}$, is easily seen to be a subalgebra of $\mathfrak{A}$. R. H. Bruck and $\mathrm{E}$. Kleinfeld [2] have recently called this subalgebra the nucleus of $\mathfrak{A}$, and we shall adopt their terminology. For an alternative algebra $\mathfrak{A},(14)$ becomes

$$
(x, y, g)=0
$$

for all $x, y$ in $\mathfrak{A}$.

In any nonassociative algebra $\mathfrak{A}$, we call the subspace $\mathfrak{P}$ which is spanned by the associators $(x, y, z)$ in $\mathfrak{A}$ the associator subspace of $\mathfrak{A}$. For any derivation $D$ we have

$$
(x, y, z) D=(x D, y, z)+(x, y D, z)+(x, y, z D) .
$$

It follows that the associator subspace $\mathfrak{B}$ of $\mathfrak{A}$ is characteristic; that is, $\mathfrak{B D} \mathfrak{B}$ for any $D$. Also the nucleus $(S)$ of any nonassociative algebra is characteristic, as may be seen by replacing $x, y, z$ in (15) in turn by $g$ in (\$).

For any $g$ in $(\mathfrak{S}$,

$$
D=R_{g}-L_{g}
$$

is a derivation of $\mathfrak{A}$, as may be verified by the same simple computation which establishes this fact for associative algebras. In the case of alternative algebras we have the converse.

LEMмA 1. If $\mathfrak{A}$ is an alternative algebra of characteristic not three, and if (16) is a derivation of $\mathfrak{A}$, then $\mathrm{g}$ is in the nucleus $\mathbb{B}$ of $\mathfrak{A}$. in $\mathfrak{A}$.

For (8) implies $(x, y, g)+(g, x, y)-(x, g, y)=3(x, y, g)=0$ for all $x, y$

We shall call any derivation of an alternative algebra $\mathfrak{A}$ which is a sum

$$
D=R_{g}-L_{g}+\sum D_{x_{i}, z_{i}}
$$

an inner derivation of $\mathfrak{A}\left({ }^{7}\right)$.

In any nonassociative algebra the identity

$$
(a b, c, d)-(a, b c, d)+(a, b, c d)=a(b, c, d)+(a, b, c) d
$$

(7) That neither (16) nor (13) may be suppressed in our definition is seen from the following examples. If $\mathfrak{A}$ is an associative, but not commutative, algebra for which $\mathfrak{Q}^{3}=\{0\}$, then every derivation (13) is 0 , while some derivation (16) is not. If $\mathfrak{A}$ is a Cayley algebra, then every derivation (16) is 0 , while every derivation of $\mathfrak{A}$ is of the form (13). 
holds $[13,(1.2)]$. Therefore, in an alternative algebra $\mathfrak{A}$, we have $(x, y z, t)$ $=-(y z, x, t)+(t, y z, x)-(x, t, y z)=2(y, z, x t)-y(z, x, t)-(y, z, x) t-t(y, z, x)$ $-(t, y, z) x-x(t, y, z)-(x, t, y) z$ by (18). Interchanging $x$ and $t$, and subtracting, we have $2(x, y z, t)=2(y, z,[x, t])-2 y(z, x, t)+2(t, x, y) z$ or, if the characteristic of $F$ is not two $\left.{ }^{8}\right)$,

$$
(x, y z, t)=(y, z,[x, t])-y(z, x, t)+(t, x, y) z .
$$

Interchange $y$ and $z$ in (19) and subtract to obtain

$$
(x,[y, z], t)=2(y, z,[x, t])-[y,(z, x, t)]+[(t, x, y), z] .
$$

Lемма 2. Let $\mathfrak{A}$ be an alternative algebra of characteristic not two, with nucleus $\$$ and associator subspace $\mathfrak{B}$. Then

$$
[x, g] \text { is in } s
$$

for all $x$ in $\mathfrak{A}, g$ in $\mathfrak{B}$, and

$$
[p, g]=0
$$

for all $p$ in $\mathfrak{B}, g$ in $\mathbb{B}$.

To obtain (21) we put $t=g$ in (19). It is sufficient to prove (22) for any associator $p=(t, x, y)$. In view of (21), this follows by putting $z=g$ in (20).

Lemma 3. Let $\mathfrak{A}$ be an alternative algebra of characteristic not two. Then $g$ in the nucleus (S) of $\mathfrak{A}$ implies

$$
\left(y^{\prime}, y^{\prime \prime}, y g\right)=\left(y^{\prime}, y^{\prime \prime}, g y\right)=\left(y^{\prime}, y^{\prime \prime}, y\right) g=g\left(y^{\prime}, y^{\prime \prime}, y\right)
$$

for all $y, y^{\prime}, y^{\prime \prime}$ in $\mathfrak{A}$.

Clearly $\left(y^{\prime}, y^{\prime \prime}, y g\right)=\left(y^{\prime}, y^{\prime \prime}, y\right) g$, and $\left(y^{\prime}, y^{\prime \prime}, g y\right)=\left(g y, y^{\prime}, y^{\prime \prime}\right)=g\left(y, y^{\prime}, y^{\prime \prime}\right)$. But $[y, g]$ in (S) by $(21)$ implies $\left(y^{\prime}, y^{\prime \prime}, y g\right)=\left(y^{\prime}, y^{\prime \prime}, g y\right)$.

The structure of semisimple alternative algebras is well known. Any such algebra is the direct sum of simple ideals which are either associative or are Cayley-Dickson algebras over their centers $[9, \S 1]\left({ }^{9}\right)$. For brevity we shall refer to any simple alternative algebra which is not associative as a Cayley algebra even though its center is not $F$.

An alternative algebra $\subseteq$ is called separable in case it is semisimple for all scalar extensions; equivalently, $\subseteq$ is a semisimple algebra in which each simple component has a separable center. It follows that if the characteristic

(8) It has subsequently come to our attention that an identity equivalent to (19) is proved in $[2,(2.9)]$. Since the proof there makes no assumption on the characteristic, formula (20) and Lemmas 2 and 3 are valid for arbitrary $F$. Also our formula (21) is implied by [2, Lemma 3.2 (i)].

(9) The center of a nonassociative algebra is the set of all elements $c$ in the nucleus for which $[x, c]=0$ for every $x$. The center is a characteristic subalgebra, and in the case of a simple alternative algebra is a field. 
of $F$ is 0 , any semisimple algebra over $F$ is separable.

Lemma 4. Let $\subseteq$ be a separable alternative algebra of characteristic not two. Then $\subseteq$ is the direct sum

$$
\mathfrak{S}=\mathfrak{B}+\mathfrak{B}
$$

of the nucleus (S) and associator subspace $\mathfrak{B}$ of $\mathfrak{S}$. If $\mathfrak{S}$ is of characteristic 0 , then $\subseteq$ is the direct sum

$$
\mathfrak{s}=3+\mathfrak{S}^{\prime}
$$

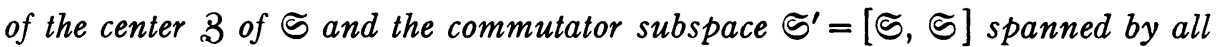
commutators $[x, y]$ in 5 .

Because $\subseteq$ is separable, we may take the base field $F$ to be algebraically closed, so that the center of each simple component is $F$. A direct sum argument reduces the proof to the case where $\subseteq$ is simple. If $\subseteq$ is associative, then (24) is trivial since $\mathbb{S}=S$ and $\mathfrak{B}=\{0\}$; also in this case (25) is well known. If $S$ is a Cayley algebra, then $\mathbb{S}=\mathbb{Z}=e F$ where $e$ is the unity element of $\mathfrak{S}$, and it is readily verified that $\mathfrak{B}=\mathfrak{S}^{\prime}$ is the subspace consisting of elements of trace 0 .

Lemma 5. Let $\mathfrak{B}$ be an alternative algebra of characteristic not two, and let $\mathfrak{S}$ be a Cayley subalgebra of $\mathfrak{B}$. If the center of $\mathfrak{E}$ is separable, then $\mathbb{E}$ is mapped into $\{0\}$ by every inner derivation of $\mathfrak{B}$ of the form (16).

We first remark that $\mathfrak{C}$ is generated by its associator subspace $\mathfrak{B}_{0}=\mathfrak{C} \cap \mathfrak{B}$. For, since $\mathfrak{C}$ is separable, it is sufficient to verify this in case $F$ is algebraically closed, in which case $\operatorname{dim} \mathfrak{E}=8=1+\operatorname{dim} \mathfrak{B}_{0}$ by (24). If $\mathfrak{B}_{0}$ does not generate $\mathfrak{E}$, then $\mathfrak{B}_{0}$ is itself a subalgebra. But then (24) implies that $\mathfrak{B}_{0}$ is an ideal of $\mathfrak{E}$, a contradiction, since $\mathfrak{S}$ is simple. Hence $\mathfrak{B}_{0}$ generates $\mathfrak{S}$, and it follows from (22) that (16) maps $\mathbb{E}$ into $\{0\}$. For we have not only $[p, g]=0$, but also $\left[p_{1} p_{2}, g\right]=p_{1}\left[p_{2}, g\right]+\left[p_{1}, g\right] p_{2}=0$, since (16) is a derivation.

Lemma 6. The radical $\mathfrak{R}$ of any alternative algebra $\mathfrak{A}$ of characteristic 0 is characteristic.

An element $z$ in $\mathfrak{A}$ is in $\mathfrak{N}$ if and only if the trace of $L_{x z}$ is zero for every $x$ in $\mathfrak{A}[1, \S 8]$. For $z$ in $\mathfrak{N}$ and any derivation $D$ of $\mathfrak{A}$, we have

$\operatorname{trace} L_{x(z D)}=\operatorname{trace} L_{(x z) D-(x D) z}=\operatorname{trace}\left[L_{x z}, D\right]-\operatorname{trace} L_{(x D) z}=0$,

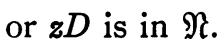

Lemma 7. Let $D$ be an inner derivation (17). Then, for all $g$ in $\$ \cap N, x_{i}$ in $\mathfrak{A}$, and $z_{i}$ in $\mathfrak{R}$, the derivation $D$ is nilpotent.

For we have shown $[9$, p. 608] that

$$
\mathfrak{N}=\mathfrak{N}^{1}>\mathfrak{N}^{2}>\cdots>\mathfrak{N}^{r}=\{0\},
$$


where each $\mathfrak{R}^{i}$ is an ideal of $\mathfrak{A}$. Let $\boldsymbol{y}$ be arbitrary in $\mathfrak{A}$. Then $y D^{i}$ is in $\mathfrak{R}^{\prime}$ for $j=1,2, \cdots$. Hence $y D^{r}=0, D^{r}=0$.

The analogue of Lemma 7 for Jordan algebras is proved in [7] for algebras of characteristic 0 as a corollary to a theorem on representations of Jordan algebras [7, Theorem 8.1]. Although we do not require the analogous theorem on representations of alternative algebras for the proof of Lemma 7, we prove the theorem below and remark that Lemma 7, for algebras of characteristic 0 , is a corollary to part (c).

Let $(S, T)$ be a representation of an alternative algebra $\mathfrak{A}$. For any subspace $\mathfrak{M}$ of $\mathfrak{A}$, we denote by $S(\mathfrak{M})$ and $T(\mathfrak{M})$ respectively the sets of all $S_{x}$ and $T_{x}$ for $x$ in $\mathfrak{M}$. We write $U(\mathfrak{M})=S(\mathfrak{M})+T(\mathfrak{M})$ for the set of all $S_{x}+T_{y}$ with $x, y$ in $\mathfrak{M}$. The respective enveloping associative algebras are denoted by $S(\mathfrak{M})^{*}, T(\mathfrak{M})^{*}, U(\mathfrak{M})^{*}$.

ThEOREM 1. Let $\mathfrak{A}$ be an alternative algebra of characteristic 0 , and $(S, T)$ be a representation of $\mathfrak{A}$. If $\mathfrak{R}$ is the radical of $\mathfrak{A}$, then

(a) $S(\mathfrak{N})$ is contained in the radical of $S(\mathfrak{U})^{*}$,

(b) $T(\mathfrak{R})$ is contained in the radical of $T(\mathfrak{H})^{*}$,

(c) $U(\mathfrak{R})$ is contained in the radical of $U(\mathfrak{R})^{*}$.

Statements (a) and (b) are immediate corollaries of [7, Theorem 8.1]. For $\mathfrak{U}^{+}$is a Jordan algebra of characteristic 0 , and $\mathfrak{R}^{+}$is its radical. Since $S$ and $T$ are Jordan representations of $\mathfrak{Q}^{+}$, we have (a) and (b). However, a separate proof must be given for (c).

The fact that $U(\mathfrak{R})^{*}$ is nilpotent may be verified by only a slight modification of the proof of [9, Lemma 1]. Formulas for $S$ and $T$ analogous to (9) and (10) imply

$$
\left[T_{x}, S_{z}\right]=-T_{[x, z]} / 2-\left[T_{x}, T_{z}\right] / 2=S_{[x, z]} / 2-\left[S_{x}, S_{z}\right] / 2 .
$$

Using the analogue of (11), we have

$$
\left[T_{y},\left[T_{x}, S_{z}\right]\right]=T_{(x, y, z)}+\left[T_{y}, S_{[x, z]}\right] .
$$

Similarly,

$$
\left[S_{y},\left[T_{x}, S_{z}\right]\right]=S_{(x, y, z)}-\left[T_{y}, S_{[x, z]}\right] .
$$

It follows that the Lie enveloping algebras of $U(\mathfrak{R})$ and $U(\mathfrak{R})$ are $\mathfrak{R}=S(\mathfrak{R})$ $+T(\mathfrak{N})+[T(\mathfrak{R}), S(\mathfrak{R})]$ and $\mathfrak{R}=S(\mathfrak{R})+T(\mathfrak{l})+[T(\mathfrak{H}), S(\mathfrak{H})]$. Let $\mathfrak{Q}=S(\mathfrak{R})$ $+T(\mathfrak{N})+[T(\mathfrak{N}), S(\mathfrak{R})]$. Since $\mathfrak{N}$ is an ideal of $\mathfrak{A}$, these identities, together with the Jacobi identity, imply that $[\mathfrak{Q}, \mathfrak{R}] \leqq \mathfrak{Q},[\Re, \mathfrak{Q}] \leqq \Re$. Hence $\mathfrak{Q}$ is an ideal in $R$ and $\Omega$ an ideal in $\mathscr{Q}$; that is, $\Re$ is subinvariant $\left({ }^{10}\right)$ in $\mathfrak{R}$. Since $\Re^{*}=U(\mathfrak{R})^{*}$, it follows from [7, Theorem 7.2] that the radical of $U(\mathfrak{R})^{*}$ contains $\Re$, and therefore also contains $U(\mathfrak{R})$ as desired.

It is clear that $S(\mathfrak{A})$ and $T(\mathfrak{R})$ are Lie triple systems [7, $\$ 1]$. However, in

(10) See $[7,87]$. 
general $U(\mathfrak{A})=S(\mathfrak{H})+T(\mathfrak{H})$ is not, as may be seen by consideration of the regular representation of a Cayley algebra.

3 . Complete reducibility. Let $(S, T)$ be a representation of an alternative algebra $\mathfrak{A}$ acting in $\mathfrak{B}$. We shall call $(S, T)$ completely reducible in case, for every invariant subspace $\mathfrak{B}$ of $\mathfrak{B}$, there is a complementary invariant subspace $\mathfrak{W}^{\prime}$ such that $\mathfrak{B}$ is the direct sum $\mathfrak{B}=\mathfrak{B}+\mathfrak{W}^{\prime}$. This is equivalent to the condition that $U(\mathfrak{A})=S(\mathfrak{A})+T(\mathfrak{A})$ be a completely reducible set of linear transformations.

The proof we give of the complete reducibility of any representation of a semisimple alternative algebra of characteristic 0 is based on the theory of completely reducible Lie algebras of linear transformations [6]. A Lie algebra of linear transformations (of characteristic 0 ) is completely reducible if and only if $\mathfrak{l}=\mathbb{R}^{\prime} \oplus \mathbb{C}$ where the derived algebra $\mathfrak{R}^{\prime}=[\mathfrak{R}, \mathbb{R}]$ is semisimple, and the center $[5$ consists of elements with simple elementary divisors [6, Theorem 1$]$. $\mathrm{N}$. Jacobson has kindly allowed us to include here the following lemma.

LEMMA 8 (JACOBSON). Let $\mathfrak{\&}$ be a Lie algebra of linear transformations of characteristic 0 such that $\mathfrak{R}=\mathfrak{M}+\mathfrak{N}$ where $\mathfrak{M}$ and $\mathfrak{N}$ are ideals of $\mathfrak{R}$ and are completely reducible. Then $\mathbb{R}$ is completely reducible.

Let $\mathfrak{E}$ be the center of $\mathfrak{R}, \mathfrak{C}_{1}$ that of $\mathfrak{M}, \mathfrak{C}_{2}$ that of $\mathfrak{R}$. We prove first that $\mathfrak{C}_{i} \leqq \mathfrak{C}$. We know that, if $C_{1}$ is in $\mathfrak{\mho}_{1}$, then $C_{1}$ has simple elementary divisors. This is equivalent to the statement that the minimum polynomial $\mu(\lambda)$ of $C_{1}$ has distinct roots. Thus $\left(\mu^{\prime}(\lambda), \mu(\lambda)\right)=1$, and $\mu^{\prime}\left(C_{1}\right)$ is nonsingular. Let $N$ be in $\mathfrak{N}$. Since $\left[\mathfrak{G}_{1}, \mathfrak{N}\right] \leqq \mathfrak{\complement}_{1}, C_{1}^{\prime} \equiv\left[C_{1}, N\right]$ commutes with $C_{1}$. Hence $\left[\mu\left(C_{1}\right), N\right]$ $=\mu^{\prime}\left(C_{1}\right) C_{1}^{\prime}=0$ and, since $\mu^{\prime}\left(C_{1}\right)$ is nonsingular, $C_{1}^{\prime}=0$. Thus $\left[\mathfrak{G}_{1}, \mathfrak{R}\right]=\{0\}$ and $\left[\mathfrak{C}_{1}, \mathfrak{R}\right]=\{0\}$. Similarly $\left[\mathfrak{C}_{2}, \mathfrak{R}\right]=\{0\}$. Next consider $\mathfrak{R}^{\prime}=\mathfrak{M}^{\prime}+[\mathfrak{M}, \mathfrak{N}]$ $+\mathfrak{N}^{\prime}$. Since $[\mathfrak{M}, \mathfrak{N}]=\left[\mathfrak{M}^{\prime}, \mathfrak{R}\right]+\left[\mathfrak{G}_{1}, \mathfrak{R}\right]=\left[\mathfrak{M}^{\prime}, \mathfrak{R}\right] \leqq \mathfrak{M}^{\prime}$, we have $\mathfrak{R}^{\prime}=\mathfrak{M}^{\prime}+\mathfrak{N}^{\prime}$. Now $\mathfrak{R}^{\prime} / \mathfrak{M}^{\prime} \cong \mathfrak{N}^{\prime} /\left(\mathfrak{M}^{\prime} \cap \mathfrak{R}^{\prime}\right)$ is semisimple and $\mathfrak{M}^{\prime}$ is semisimple. Hence $\mathfrak{R}^{\prime}$ is semisimple. Next $\mathfrak{R}=\mathfrak{M}+\mathfrak{N}=\left(\mathfrak{M}^{\prime}+\mathfrak{N}^{\prime}\right)+\left(\mathfrak{C}_{1}+\mathfrak{V}_{2}\right)=\mathfrak{R}^{\prime}+\left(\mathfrak{E}_{1}+\mathfrak{C}_{2}\right)$ and $\mathfrak{l}^{\prime}$ $\cap\left(\mathfrak{S}_{1}+\mathfrak{C}_{2}\right)=\{0\}$. It follows that $\mathfrak{夭}_{1}+\mathfrak{C}_{2}=\mathfrak{C}$. If $C_{1}$ is in $\mathfrak{夭}_{1}, C_{2}$ in $\mathfrak{E}_{2}$, then $C_{1}+C_{2}$ has simple elementary divisors ${ }^{(11)}$. Thus every element of $\mathbb{E}$ has simple elementary divisors, and this completes the proof.

THEOREM 2. Let $\mathfrak{A}$ be a semisimple alternative algebra of characteristic 0. Then any representation $(S, T)$ of $\mathfrak{A}$ is completely reducible.

We need to show that $U(\mathfrak{A})=S(\mathfrak{A})+T(\mathfrak{A})$ is a completely reducible set of linear transformations. We do this by showing that the enveloping Lie algebra $\mathfrak{R}$ of $U(\mathfrak{R})$ is a completely reducible Lie algebra.

If $\mathfrak{R}_{S}$ and $\mathfrak{R}_{T}$ are the Lie enveloping algebras $\mathfrak{R}_{S}=S(\mathfrak{U})+[S(\mathfrak{H}), S(\mathfrak{U})]$,

(11) A short proof of this well known fact may be given as follows. The algebras $F\left[C_{1}\right]$ and $F\left[C_{2}\right]$ are semisimple. The algebra $F\left[C_{1}, C_{2}\right]$ is a homomorphic image of $F\left[C_{1}\right] \times F\left[C_{2}\right]$, and the latter is semisimple. Hence $F\left[C_{1}, C_{2}\right]$ is semisimple, and all of its elements have simple elementary divisors. 
$\mathfrak{R}_{T}=T(\mathfrak{A})+[T(\mathfrak{A}), T(\mathfrak{A})]$ of $S(\mathfrak{U})$ and $T(\mathfrak{A})$ respectively, then $(27)$, with the Jacobi identity, implies that $\mathfrak{R}=\mathfrak{R}_{S}+\mathfrak{R}_{T}$ where $\mathfrak{R}_{S}$ and $\mathfrak{R}_{T}$ are ideals of $\mathfrak{R}$. Since $S$ and $T$ are (Jordan) representations of the semisimple Jordan algebra $\mathfrak{A}^{+}$, we know that $\mathfrak{\Omega}_{S}$ and $\mathfrak{R}_{T}$ are completely reducible [7, Corollary 8.1]. The theorem follows from Lemma 8 by setting $\mathfrak{N}=\mathfrak{R}_{S}, \mathfrak{N}=\mathfrak{R}_{T}$.

REMARK. Jacobson has observed that the universal associative algebra of the representations of an alternative algebra is finite-dimensional $[7, \S \S 3,6]$. This simply means that the dimensionalities of the enveloping algebras of the representations have a fixed upper bound. One can see this by observing that, if $u_{1}, u_{2}, \cdots, u_{n}$ is a basis of $\mathfrak{A}$. then any element of the enveloping associative algebra $\xi=U(\mathfrak{U}) *$ is a linear combination of elements $S_{u_{1}}^{\epsilon_{1}} S_{u_{2}}^{\epsilon_{2}} \ldots$ $S_{u_{n}}^{\epsilon_{n}} T_{u_{1}}^{\epsilon_{n+1}} \cdots T_{u_{n}}^{\epsilon_{2 n}}, \epsilon_{i}=0,1$. This follows easily from $S_{x} S_{z}+S_{z} S_{x}=S_{x z+z x}$, $T_{x} T_{z}+T_{z} T_{x}=T_{x z+z x}, S_{x} T_{z}-T_{z} S_{x}=S_{x z}-S_{x} S_{z}$. Thus dim $\xi \leqq 2^{2 n}$. This has the usual consequence that there are only a finite number of inequivalent irreducible representations.

4. The first Whitehead lemma. We begin by proving a theorem for alternative algebras which is a generalization of the first Whitehead lemma for associative algebras [5, Theorem 2.2]. Our theorem is a corollary to the analogous theorem for Jordan algebras [7, Theorem 9.1].

THEOREM 3. Let $\mathfrak{A}$ be a semisimple alternative algebra of characteristic 0 with representation $(S, T)$ acting in $\mathfrak{B}$. Let $\nu$ be a 1-cocycle of $\mathfrak{A}$, that is, a linear mapping of $\mathfrak{A}$ into $\mathfrak{B}$ such that $\nu(x y)=x \nu(y)+\nu(x) y=\nu(y) T_{x}+\nu(x) S_{y}$ holds for all $x, y$ in $\mathfrak{A}$. Then, if $\mathfrak{B}$ is the semidirect sum $\mathfrak{B}=\mathfrak{A}+\mathfrak{B}$, there exist elements $x_{i}, x_{i}^{\prime}$ in $\mathfrak{A}, z_{i}, z_{i}^{\prime}$ in $\mathfrak{B}$, such that

$$
g=\sum\left[x_{i}^{\prime}, z_{i}^{\prime}\right]
$$

in $\mathfrak{B}$ is in the nucleus $\mathbb{S}$ of $\mathfrak{B}$, and

$$
\nu(y)=[y, g]+\sum y D_{x_{i}, z_{i}}=y D
$$

for every $y$ in $\mathfrak{A}$, where $D$ is the inner derivation (17) of $\mathfrak{B}$.

For proof, we observe that $\mathfrak{A}^{+}$is a semisimple Jordan algebra and that $S^{+}$ defined by $(7)$ is a Jordan representation of $\mathfrak{A}^{+}$acting in $\mathfrak{B}^{+}$. Moreover, $\nu(x \cdot y)=\nu(x y)+\nu(y x)=x \cdot \nu(y)+\nu(x) \cdot y$, so that $\nu$ is a mapping satisfying the requirements of the first Whitehead lemma for Jordan algebras. Hence there exist elements $a_{i}$ in $\mathfrak{A}$ and $v_{i}$ in $\mathfrak{B}$ such that

$$
\begin{aligned}
\nu(y) & =\sum y\left[R_{a_{i}}^{+}, R_{v_{i}}^{+}\right]=\sum y\left[R_{a_{i}}+L_{a_{i}}, R_{v_{i}}+L_{v_{i}}\right] \\
& =\sum y\left\{D_{a_{i}, v_{i}}+\left[L_{a_{i}}, R_{v_{i}}\right]\right\} \\
& =\frac{1}{3} \sum y\left\{R_{\left[a_{i}, v_{i}\right]}-L_{\left[a_{i}, v_{i}\right]}+2 D_{a_{i}, v_{i}}\right\}
\end{aligned}
$$


by (12). That is, (29) holds for $g$ in (28) where $x_{i}^{\prime}=(1 / 3) a_{i}, x_{i}=(2 / 3) a_{i}$, $z_{i}^{\prime}=z_{i}=v_{i}$. Now the linear transformation $D$ on $\mathscr{B}$ defined by $v D=0$ for $v$ in $\mathfrak{B}$ and $y D=\nu(y)$ for $y$ in $\mathfrak{A}$ is a derivation of $\mathfrak{B}$. Also $D$ has the form (17) except that it has not yet been proved that $g$ is in 3 . However, $D-\sum D_{x_{i}, z_{i}}$ $=R_{g}-L_{g}$ is a derivation of $\mathscr{B}$ since $D$ and $\sum D_{x_{i}, z_{i}}$ are. It follows from Lemma 1 that $g$ is in (S).

To see that Theorem 3 includes the first Whitehead lemma for associative algebras, we observe that, if $\mathfrak{A}$ is associative and if $\mathfrak{B}$ is a two-sided $\mathfrak{A}$-module, then the semidirect sum $\mathfrak{B}=\mathfrak{A}+\mathfrak{B}$ is associative, and $D_{x_{i}, z_{i}}$ in (29) becomes $R_{\left[x_{i}, z_{i}\right]}-L_{\left[x_{i}, z_{i}\right]}$ because the last term of (12) vanishes. Thus $\nu(y)=\left[y, g^{\prime}\right]$ where $g^{\prime}=g+\sum\left[x_{i}, z_{i}\right]=\sum\left[a_{i}, v_{i}\right]$ is in $[\mathfrak{A}, \mathfrak{B}] \leqq \mathfrak{B}$.

In the proof of the Malcev theorem in the next section, we shall require a stronger form of the first Whitehead lemma for alternative algebras.

THEOREM 4. Let the hypotheses be as in Theorem 3. Then we may take $g=0$ in (29) so that

$$
\nu(y)=\sum y D_{x_{i}, z_{i}}
$$

for $x_{i}$ in $\mathfrak{A}, z_{i}$ in $\mathfrak{B}$.

Since $(S, T)$ is completely reducible by Theorem 2 , it may be expressed in a natural way as a sum of irreducible representations acting in invariant subspaces of $\mathfrak{B}$. Also $\nu$ may be expressed as the sum of corresponding mappings into these subspaces. Therefore we may assume that the given representation $(S, T)$ is irreducible.

It is sufficient to show either that

$$
[y, g]=0
$$

for every $y$ in $\mathfrak{A}$, or that

$$
\sum\left(x_{i}^{\prime}, y, z_{i}^{\prime}\right)=0
$$

for every $y$ in $\mathfrak{A}$ (where $x_{i}^{\prime}, z_{i}^{\prime}$ are as in Theorem 3). The sufficiency of (31) is obvious. On the other hand, (32) implies that $\sum\left(3 x_{i}^{\prime}, y, z_{i}^{\prime}\right)=\sum\left(a_{i}, y, v_{i}\right)$ $=\sum y\left[L_{a_{i}}, R_{v_{i}}\right]=0$ so $\nu(y)=\sum y\left\{D_{a_{i}, v_{i}}+\left[L_{a_{i}}, R_{v_{i}}\right]\right\}=\sum y D_{a_{i}, v_{i}}$ in the desired form.

Let $\mathfrak{A}=\mathfrak{S}_{1} \oplus \cdots \oplus \mathfrak{S}_{t}$ for simple $\mathfrak{S}_{j}$, and let $e_{j}$ be the unity element of $\mathfrak{S}_{j}$. Consider the Peirce decomposition of $\mathfrak{B}$ relative to the pairwise orthogonal idempotents $e_{j}, j=1, \cdots, t$. We have $\mathfrak{B}$ the direct sum

$$
\mathfrak{B}=\sum_{i, k=0}^{\mathfrak{t}} \mathfrak{B}_{i k}
$$

where $v_{i k}$ is in $\mathfrak{B}_{i k}$ if and only if

$$
v_{i k} \text { is in } \mathfrak{B}, \quad e_{j} v_{i k}=\delta_{j i} v_{i k}, \quad v_{i k} e_{j}=\delta_{k j} v_{i k},
$$


(Kronecker delta). The properties of a Peirce decomposition $[12, \S 2 ; 13, \S 4]$ readily imply that

$$
\left(e_{j}, x, z\right)=0
$$

for $x$ in $\mathfrak{A}, z$ in $\mathfrak{B}$, and

$$
\left(x_{j}, x_{i}, z\right)=0
$$

for $x_{j}$ in $\mathfrak{S}_{j}, x_{i}$ in $\mathfrak{S}_{i}, z$ in $\mathfrak{B}$. Using (33) and (34), it is easily verified that, since $\mathfrak{B}^{2}=\{0\}$, each of the subspaces $\mathfrak{B}_{i k}$ is an ideal of $\mathfrak{B}$. The irreducibility of the representation $(S, T)$ is equivalent to the assumption that there are no nonzero ideals of $\mathfrak{B}$ properly contained in $\mathfrak{B}$. Hence all but one of the $\mathfrak{B}_{i k}$ are zero, and

$$
\mathfrak{B}=\mathfrak{B}_{i k}
$$

for some fixed pair of subscripts $i, k(0 \leqq i, k \leqq t)$.

In case $i=0$ or $k=0$, we have $(x, y, z)=0$ trivially for $x, y$ in $\mathfrak{A}, z$ in $\mathfrak{B}$. For example, if $i=0$, then $z=v_{0 k}$ implies $(x, y, z)=\left\{(x y) \sum e_{j}\right\} v_{0 k}$ $-x\left\{\left(y \sum e_{j}\right) v_{0 k}\right\}=0$ by (34) and the fact that $e_{j} v_{0 k}=0$ for $j=1, \cdots, t$. Therefore, in case $i=0$ or $k=0,(32)$ is satisfied, and we may take $\mathfrak{B}=\mathfrak{B}_{i k}$ for some fixed pair $i, k(1 \leqq i, k \leqq t)$.

We shall now show that, in case either $\mathfrak{S}_{i}$ or $\mathfrak{S}_{k}$ is associative, the intersection $\mathfrak{S}_{0}=\mathfrak{B} \cap \mathfrak{B}$ of $\mathfrak{B}$ and the nucleus $\mathfrak{S}$ of $\mathfrak{B}$ is an ideal of $\mathfrak{B}$. For elements $g=g_{\text {ik }}$ in $\mathfrak{S}_{0} \leqq \mathfrak{B}=\mathfrak{B}_{i k}$ and $b=y+v$ in $\mathfrak{B}$ ( $y$ in $\mathfrak{A}$, $v$ in $\mathfrak{B}$ ), we have $b g$ and $g b$ in $\mathfrak{B}$ and shall show that $\left(b^{\prime}, b^{\prime \prime}, b g\right)=\left(b^{\prime}, b^{\prime \prime}, g b\right)=0$. Write $y=\sum y_{j}, y_{j}$ in $\varsigma_{j}$. Since $\mathfrak{B}^{2}=\{0\},(23)$ and (35) imply $\left(b^{\prime}, b^{\prime \prime}, b g\right)=\left(b^{\prime}, b^{\prime \prime}, g b\right)=\left(y^{\prime}, y^{\prime \prime}, g y\right)$ $=\left(y^{\prime}, y^{\prime \prime}, y\right) g_{i k}=\sum\left(y_{h}^{\prime}, y_{i}{ }^{\prime \prime}, y_{j}\right) g_{i k}=\sum\left(y_{j}^{\prime}, y_{j}^{\prime \prime}, y_{j}\right) g_{i k}=\sum\left(y_{j}^{\prime}, y_{j}^{\prime \prime}, y_{j}\right) e_{j} g_{i k}$ $=\left(y_{i}^{\prime}, y_{i}^{\prime \prime}, y_{i}\right) g_{i k}=0$ in case $\mathfrak{S}_{i}$ is associative, while similarly $\left(b^{\prime}, b^{\prime \prime}, b g\right)$ $=\left(b^{\prime}, b^{\prime \prime}, g b\right)=g_{i k}\left(y^{\prime}, y^{\prime \prime}, y\right)=g_{i k}\left(y_{k}^{\prime}, y_{k}^{\prime \prime}, y_{k}\right)=0$ in case $\Im_{k}$ is associative. Hence, in either case, $b g$ and $g b$ are in $\mathscr{S}_{0}=\mathfrak{B} \cap \mathscr{B}$, and $\mathfrak{H}_{0}$ is an ideal of $\mathfrak{B}$. By the irreducibility of the representation, either $\mathfrak{B S}_{0}=\mathfrak{B}$ or $\mathfrak{B}_{0}=\{0\}$. In the first case (32) holds; in the second, $g=0$ implies (31).

There remains the case where neither $\mathfrak{S}_{i}$ nor $\mathfrak{S}_{k}$ is associative; that is, each is a Cayley subalgebra of $\mathfrak{B}$. Compute $[y, g]=\left[y, g_{i k}\right]=\sum\left[y_{j}, g_{i k}\right]=y_{i} g_{i k}$ $-g_{i k} y_{k}$. We have

$$
[y, g]= \begin{cases}{\left[y_{i}, g_{i k}\right]+\left[y_{k}, g_{i k}\right]} & \text { if } i \neq k, \\ {\left[y_{i}, g_{i i}\right]} & \text { if } i=k .\end{cases}
$$

But $\left[y_{i}, g\right]=\left[y_{k}, g\right]=0$ by Lemma 5 . Then (36) gives (31), completing the proof.

As in $[5$, Theorems 3.1, 3.2] it is readily seen that Theorem 4 is equivalent to

THEOREM 5. If $\mathfrak{A}$ is a semisimple subalgebra of an alternative algebra $\mathfrak{B}$ of 
characteristic 0 , then any derivation of $\mathfrak{A}$ into $\mathfrak{B}$ can be extended to an inner derivation (13) of $\mathfrak{B}$.

5. The Malcev theorem. We have shown in [9] that the Wedderburn principal theorem for associative algebras generalizes to alternative algebras over an arbitrary field $F$. That is, if $\mathfrak{A}$ is an alternative algebra with radical $\mathfrak{N}$, and if $\mathfrak{A} / \mathfrak{R}$ is separable, then $\mathfrak{A}$ may be expressed as the direct sum

$$
\mathfrak{A}=\mathfrak{s}+\mathfrak{N},
$$

where $\subseteq$ is a separable subalgebra of $\mathfrak{A}$ isomorphic to $\mathfrak{A} / \mathfrak{R}$.

The subalgebra $\mathfrak{S}$ in the Wedderburn decomposition (37) need not be unique but if $\mathfrak{A}=\mathfrak{S}_{1}+\mathfrak{N}$ is another such decomposition, then Malcev has shown that, in case $\mathfrak{A}$ is associative, $\mathfrak{S}_{1}$ can be mapped onto $\subseteq$ by an inner automorphism of $\mathfrak{A}$ of a special type [8,Theorem 2]. We shall generalize his result to the case of alternative algebras, but only for those of characteristic 0 . For, following the proofs of Harish-Chandra [4] and Jacobson [7] for Lie and Jordan algebras, we base the proof of this theorem on the Whitehead first lemma. The automorphism in question has the form (38) below $\left.{ }^{(12}\right)$.

If $\mathfrak{A}$ is a nonassociative algebra of characteristic 0 , and if $D$ is a nilpotent derivation of $\mathfrak{A}$, then

$$
G=\exp D=I+D+\frac{D^{2}}{2 !}+\frac{D^{3}}{3 !}+\cdots
$$

is an automorphism of $\mathfrak{A}$. Two subalgebras of $\mathfrak{A}$ are called strictly conjugate $[7, \S 9]$ if one is mapped onto the other by an automorphism of the form $G_{1} G_{2} \cdots G_{k}, G_{i}=\exp D_{i}, D_{i}$ a nilpotent derivation.

THEOREM 6. Let $\mathfrak{A}$ be an alternative algebra of characteristic 0 with Wedderburn decomposition $\mathfrak{A}=\mathfrak{S}+\mathfrak{R}$, and let $\mathfrak{M}$ be a semisimple subalgebra of $\mathfrak{A}$. Then $\mathfrak{M}$ is strictly conjugate to a subalgebra of $\subseteq$ under an automorphism $G$ $=\exp D$ of $\mathfrak{A}$ where $D$ is a derivation of $\mathfrak{A}$ and $D$ is in the radical $\Re$ of the enveloping associative algebra of the right and left multiplications of $\mathfrak{A}$.

The proof is essentially the same as those for Lie and Jordan algebras [4, Theorem $2 ; 7$, Theorem 9.3]. We prove by induction on $k$ that there is an automorphism $G^{\prime}=\exp D^{\prime}$ of $\mathfrak{A}$ mapping $\mathfrak{M}$ into $\mathfrak{S}+\mathfrak{N}^{k}$ for $D^{\prime}$ in $\Re$. The case $k=1$ is given by $D^{\prime}=0$. By (26) the case $k=r$ establishes the theorem. We assume that $\widetilde{G}=\exp \widetilde{D}, \widetilde{D}$ in $\Re$, maps $\mathfrak{M}$ onto $\mathfrak{M}_{k} \leqq \subseteq ্ \mathfrak{N}^{k}$, and shall show that there exists $D$ in $\Re$ such that $G=\exp D$ maps $\mathfrak{M}_{k}$ into $\mathfrak{S}+\mathfrak{R}^{k+1}$. The

(12) Note that, in case $\mathfrak{A}$ is associative and of characteristic 0 , the automorphism $G$ $=\exp \left(R_{z}-L_{z}\right)$ for $z$ in $\mathfrak{N}$ reduces to Malcev's inner automorphism $a \rightarrow a-a x-x^{\prime} a+x^{\prime} a x$ generated by an element $x$ in $\mathfrak{N}$ (where $x^{\prime}$, the quasi-inverse of $x$, is the unique solution of $x x^{\prime}=x^{\prime} x$ $\left.=x+x^{\prime}\right)$. For we may take $x=-z-z^{2} / 2 !-z^{3} / 3 !-\cdots$ in $\mathfrak{R}$ and $x^{\prime}=-x-x^{2}-x^{3}-\cdots$ $=z-z^{2} / 2 !+z^{3} / 3 !-\cdots$. 
Campbell-Hausdorff formula

$$
\exp \widetilde{D} \exp D=\exp (\tilde{D}+D+(1 / 2)[\tilde{D}, D]+\cdots)
$$

implies that $G^{\prime}=\widetilde{G} G=\exp D^{\prime}$ for $D^{\prime}$ in $\Re$ as required.

Every $x$ in $\mathfrak{M}_{k}$ may be written uniquely in the form $x=\sigma(x)+\nu(x)$ where $\sigma(x)$ is in $\subseteq$ and $\nu(x)$ is in $\mathfrak{N}^{k}$. The mappings $x \rightarrow \sigma(x)$ and $x \rightarrow \nu(x)$ are linear. Also $x y=\sigma(x y)+\nu(x y)=\sigma(x) \sigma(y)+\sigma(x) \nu(y)+\nu(x) \sigma(y)+\nu(x) \nu(y)$ so that

$$
\sigma(x y)=\sigma(x) \sigma(y)
$$

and

$$
\nu(x y)=\sigma(x) \nu(y)+\nu(x) \sigma(y)+\nu(x) \nu(y) .
$$

Since $\sigma$ is a homomorphism of $\mathfrak{M}_{k}$ into $\subseteq$ by (39) and the $\mathfrak{R}^{i}$ are ideals of $\mathfrak{A}$, the mappings $x \rightarrow R_{\sigma(x)}, x \rightarrow L_{\sigma(x)}$ induce a representation $(S, T)$ of $\mathfrak{M}_{k}$ acting in $\mathfrak{N}^{k} / \mathfrak{N}^{k+1}$. Write $\bar{a}, \bar{\sigma}(x), \bar{\nu}(y)$ for the residue classes modulo $\mathfrak{R}^{k+1}$ of $a, \sigma(x)$, $\nu(y)$ respectively. Then $(S, T)$ is defined by

$$
\bar{z} x=\bar{z} S_{x}=\bar{z} \bar{\sigma}(x), \quad x \bar{z}=\bar{z} T_{x}=\bar{\sigma}(x) \bar{z}
$$

for $\bar{z}$ in $\mathfrak{\Re}^{k} / \mathfrak{\Re}^{k+1}, x$ in $\mathfrak{M}_{k}$.

Since $\left(\mathfrak{R}^{k} / \mathfrak{R}^{k+1}\right)^{2}=\{0\}$, it follows from (40) that the mapping $y \rightarrow \bar{\nu}(y)$ is a 1-cocycle of $\mathfrak{M}_{k}$. By Theorem 4 there exist elements $x_{i}$ in $\mathfrak{M}_{k}, \bar{z}_{i}$ in $\mathfrak{R}^{k} / \mathfrak{N}^{k+1}$ such that $\bar{\nu}(y)=\sum y D_{x_{i}, \bar{z}_{i}}$ so that

$$
\nu(y) \equiv \sum \sigma(y) D_{\sigma\left(x_{i}\right), z_{i}}
$$

$\left(\bmod \mathfrak{N}^{k+1}\right)$

for $x_{i}$ in $\mathfrak{M}_{k}, \boldsymbol{z}_{i}$ in $\mathfrak{R}^{k}$. The mapping $D=-\sum D_{\sigma\left(x_{i}\right), z_{i}}$ is a nilpotent derivation of $\mathfrak{A}$ by Lemma 7 ; hence $G=\exp D$ is an automorphism. Actually $D$ is in $\Re$ by Theorem 1 (c). For $y$ in $\mathfrak{M}_{k}$, we have $y G=\sigma(y) G+\nu(y) G=\sigma(y)+\sigma(y) D$ $+\cdots+\nu(y)+\cdots$, where the terms denoted by $\cdots$ belong to $\mathfrak{N}^{k+1}$. Hence $y G \equiv \sigma(y)+\sigma(y) D+\nu(y) \equiv \sigma(y)\left(\bmod \mathfrak{R}^{k+1}\right)$ by $(41)$, or $\mathfrak{M}_{k} G \leqq \subseteq+\mathfrak{R}^{k+1}$ as desired.

Corollary 1 (The Malcev Theorem). If $\mathfrak{A}$ has Wedderburn decompositions $\mathfrak{A}=\mathfrak{S}+\mathfrak{N}=\mathfrak{S}_{1}+\mathfrak{N}$, then $\mathfrak{S}_{1}$ is strictly conjugate to $\mathfrak{S}$ under an automorphism $G=\exp D, D$ in $\Re$.

CoRollary 2. Any semisimple subalgebra of $\mathfrak{A}$ can be imbedded in the semisimple component of some Wedderburn decomposition of $\mathfrak{A}$.

We needed the strong form of the first Whitehead lemma because, if we had taken $\bar{\nu}(y)=\left[\begin{array}{ll}y, & \bar{g}\end{array}\right]+\sum y D_{x_{i}, z_{i}}$, we would have $\nu(y) \equiv[\sigma(y), g]$ $+\sum \sigma(y) D_{\sigma\left(x_{i}\right), z_{i}}\left(\bmod \mathfrak{R}^{k+1}\right)$ for a representative $g$ of $\bar{g}$, where $\bar{g}$ is in the nucleus of $\overline{\mathfrak{A}}$. But there is no guarantee that $g$ may be chosen in the nucleus of $\mathfrak{A}$. If we cannot do this, $R_{g}-L_{g}$ is not a derivation. On the other hand, $\sum D_{\sigma\left(x_{i}\right), z_{i}}$ is always a derivation of $\mathfrak{A}$.

For example, consider the alternative algebra $\mathfrak{A}$ with basis $e, z_{1}, z_{2}, z_{3}$ and 
multiplication table

\begin{tabular}{c|cccc} 
& $e$ & $z_{1}$ & $z_{2}$ & $z_{3}$ \\
\hline$e$ & $e$ & $z_{1}$ & $z_{2}$ & 0 \\
$z_{1}$ & 0 & 0 & $z_{3}$ & 0 \\
$z_{2}$ & 0 & $-z_{3}$ & 0 & 0 \\
$z_{3}$ & $z_{3}$ & 0 & 0 & 0
\end{tabular}

The radical of $\mathfrak{A}$ is $\mathfrak{N}=\left(z_{1}, z_{2}, z_{3}\right)$ and $\mathfrak{A}=e F+\mathfrak{N}$ is a Wedderburn decomposition of $\mathfrak{A}$. The nucleus of $\mathfrak{A}$ is $\mathfrak{R}^{2}=z_{3} F$. Now $\overline{\mathfrak{A}}=\mathfrak{A} / \mathfrak{N}^{2}$ is associative. Hence $\bar{z}_{1}$ in $\overline{\mathfrak{R}}=\mathfrak{N} / \mathfrak{N}^{2}$ is in the nucleus of $\overrightarrow{\mathfrak{l}}$, but there is no $g$ in $\bar{z}_{1}$ such that $g$ is in the nucleus of $\mathfrak{A}$.

It is to circumvent this difficulty with the passage from the nucleus of $\overline{\mathfrak{A}}$ to the nucleus of $\mathfrak{A}$ that we have established Theorem 4 . This would be unnecessary in case $\mathfrak{R}^{2}=\{0\}$, for then (29) suffices for the proof.

6. A theorem on derivation algebras. The set $\mathfrak{D}$ of all derivations of a nonassociative algebra $\mathfrak{A}$ is a Lie algebra called the derivation algebra of $\mathfrak{A}$, multiplication in $\mathfrak{D}$ being given by $\left[D_{1}, D_{2}\right]=D_{1} D_{2}-D_{2} D_{1}$. Hochschild has shown that any associative algebra $\mathfrak{A}$ of characteristic 0 is semisimple if and only if its derivation algebra $\mathfrak{D}$ is semisimple or $\{0\}$ [5, Theorem 4.5]. We generalize this theorem to alternative algebras $\mathfrak{A}$. Corresponding theorems for Lie and Jordan algebras are known [5, Theorem $4.4 ; 11]$.

Lemma 9. Let $\mathfrak{A}$ be an alternative algebra of characteristic 0 with radical $\mathfrak{\Re}$. Then the identity,

$$
D_{x, z}=R_{[x, z]}-L_{[x, z]}-3\left[L_{x}, R_{z}\right]=0
$$

for all $x$ in $\mathfrak{A}$ and all $\boldsymbol{z}$ in $\mathfrak{N}$, implies that $\mathfrak{N}$ is contained in the nucleus of $\mathfrak{A}$.

In any alternative algebra $\mathfrak{A}$ we have the identity $[12$, p. 126]

$$
[y,[x, z]]+[x,[z, y]]+[z,[y, x]]=6(x, y, z) .
$$

Now (42) implies, for all $x, y$ in $\mathfrak{A}$ and $z$ in $\mathfrak{N}$, the first equality in

$$
[y,[x, z]]=3(x, y, z)=[x,[z, y]] \text {. }
$$

The second equality follows by interchange of $x$ and $y$. It follows from (43) that, for all $x, y$ in $\mathfrak{A}, z$ in $\mathfrak{N}$,

$$
[z,[y, x]]=0 .
$$

Let $\mathfrak{A}=\Im+\mathfrak{N}$ be a Wedderburn decomposition of $\mathfrak{A}$, and write $x=s_{1}+z_{1}$, $y=s_{2}+z_{2}$ for $s_{i}$ in $\mathfrak{S}_{,}, z_{i}$ in $\mathfrak{R}$. Then $[y,[x, z]]=\left[s_{2},\left[s_{1}, z\right]\right]+\left[s_{2},\left[z_{1}, z\right]\right]$ by (45). However, $\left[s_{2},\left[z_{1}, z\right]\right]=\left[z,\left[s_{2}, z_{1}\right]\right]=0$ by (44) and (45). Hence $3(x, y, z)$ $=[y,[x, z]]=\left[s_{2},\left[s_{1}, z\right]\right]$. Now Lemma 4 implies that $s_{i}=c_{i}+\sum\left[t_{i}, t_{i}^{\prime}\right]$ for $c_{i}$ in the center $\mathbb{B}$ of $\mathfrak{S}$. Then $\left[s_{1}, z\right]=\left[c_{1}, z\right]+\sum\left[\left[t_{1}, t_{1}^{\prime}\right], z\right]=\left[c_{1}, z\right]$ by $(45)$, 
while $\left[\left[t_{2}, t_{2}^{\prime}\right],\left[c_{1}, z\right]\right]=\left[c_{1},\left[z,\left[t_{2}, t_{2}^{\prime}\right]\right]\right]=0$ by $(44)$ and $(45)$. Hence $3(x, y, z)$ $=\left[s_{2},\left[s_{1}, z\right]\right]=\left[c_{2},\left[c_{1}, z\right]\right]=3\left(c_{1}, c_{2}, z\right)$.

Let $\Omega$ be the algebraic closure of the base field. Since $\mathfrak{A}$ is of characteristic 0 ; the radical of $\mathfrak{A}_{\Omega}$ is $\mathfrak{R}_{\Omega}$. Thus, it is sufficient to prove that $\left(c_{1}, c_{2}, z\right)$ $=0$ in case the base field is algebraically closed. But then any $c$ in 8 is a linear combination of pairwise orthogonal idempotents $e_{j}$, and $(x, y, z)$ $=\left(c_{1}, c_{2}, z\right)$ is a linear combination of associators $\left(e_{i}, e_{j}, z\right)$ which are all zero $[12, \S 2]$. Hence $(x, y, z)=0$ for all $x, y$ in $\mathfrak{A}, z$ in $\mathfrak{R}$.

THEOREM 7. Let $\mathfrak{A}$ be an alternative algebra of characteristic 0 , and $\mathfrak{D}$ be its derivation algebra. Then $\mathfrak{A}$ is semisimple if and only if $\mathfrak{D}$ is semisimple or $\{0\}$.

If $\mathfrak{A}$ is semisimple, then $\mathfrak{D}$ is the direct sum of the derivation algebras of the simple components of $\mathfrak{A}$. The associative components contribute a summand which is either semisimple or $\{0\}$. Moreover, the derivation algebra of each Cayley component is simple, being an exceptional simple Lie algebra of type $G$ over the center of the Cayley component. Hence $\mathfrak{D}$ is either semisimple or $\{0\}$.

Conversely, suppose that $\mathfrak{D}$ is either semisimple or $\{0\}$. Let $\mathfrak{I}_{\mathfrak{N}}$ be the subspace of $\mathfrak{D}$ spanned by all inner derivations of $\mathfrak{A}$ of the form (12), $x$ in $\mathfrak{A}$, $z$ in $\mathfrak{R}$. It follows from the Jacobi identity that

$$
\left[D_{x, z}, D\right]=D_{x, z D}+D_{x D, z}
$$

for any derivation $D$ of $\mathfrak{A}$. Since $\mathfrak{N}$ is characteristic by Lemma $6, \mathfrak{I}_{\mathfrak{N}}$ is an ideal of $\mathfrak{D}$. Moreover, since $\mathfrak{R}$ is nilpotent, $\mathfrak{I}_{\mathfrak{N}}$ is solvable. Then, $\mathfrak{D}$ being semisimple or $\{0\}$, we have $\mathfrak{I}_{\mathfrak{N}}=\{0\}$, or (42) holds for all $x$ in $\mathfrak{A}$ and $z$ in $\mathfrak{R}$. By Lemma 9 , the nucleus $\mathbb{B}$ of $\mathfrak{A}$ contains $\mathfrak{R}$. Therefore $R_{z}-L_{z}$ is a derivation of $\mathfrak{A}$ for every $z$ in $\mathfrak{R}$. Let $\mathfrak{I}_{\mathfrak{N}}$ now be the set of all $R_{z}-L_{z}$ with $z$ in $\mathfrak{N}$. As before, $\mathfrak{D}_{\mathfrak{N}}$ is a solvable ideal of $\mathfrak{D}$, and therefore is $\{0\}$. Hence

$$
R_{z}-L_{z}=0
$$

for all $z$ in $\mathfrak{R}$.

But $\mathfrak{N} \leqq(S)$ and (46) together express the fact that $\mathfrak{R}$ is contained in the center $\mathfrak{S}$ of $\mathfrak{A}$.

Let $\mathfrak{A}=\mathfrak{S}+\mathfrak{N}$ be a Wedderburn decomposition of $\mathfrak{A}$. Then $\mathfrak{E}$ is the direct sum

$$
\mathfrak{C}=3+\mathfrak{N}
$$

where 3 is the center of $\subseteq$ and, by Lemma $4, \mathfrak{A}$ is the direct sum

$$
\mathfrak{A}=\mathfrak{S}^{\prime}+\mathfrak{c}
$$

where $\mathfrak{S}^{\prime}$ is the commutator subspace of $\mathfrak{S}$. For $z$ in $\mathfrak{N} \leqq \mathfrak{S}$, we have $z\left[a_{1}, a_{2}\right]$ $=\left[z a_{1}, a_{2}\right]=0$ for $a_{i}$ in $\mathfrak{A}$, since $z a_{1}$ is in $\mathfrak{R}$. Hence

$$
\mathfrak{N \Im ^ { \prime }}=\{0\}, \quad \mathfrak{S} \Im^{\prime} \leqq 3 \Im^{\prime} \leqq \Im^{\prime} .
$$


Similarly,

$$
\mathfrak{S}^{\prime} \mathfrak{R}=\{0\}, \quad \mathbb{S}^{\prime} \mathbb{S} \leqq \mathfrak{S}^{\prime} .
$$

Let $D_{\mathfrak{c}}$ be any derivation of the associative commutative algebra $\mathfrak{c}$. The linear transformation $D$ on $\mathfrak{A}$ defined by

$$
s^{\prime} D=0, \quad c D=c D_{\mathfrak{G}}
$$

for $s^{\prime}$ in $\mathfrak{S}^{\prime}, c$ in $\mathfrak{C}$, is a derivation of $\mathfrak{A}$, as may be checked, using (47) and (48), by the same computation as that given in the proof of the corresponding theorem for Jordan algebras [11, p. 294]. The remainder of the proof is identical with the Jordan proof: since $\mathbb{E}$ is characteristic, any derivation $D$ of $\mathfrak{A}$ induces a derivation $D_{\mathfrak{C}}$ on $\mathfrak{C}$. Thus $D \rightarrow D_{\mathfrak{C}}$ is a homomorphism of $\mathfrak{D}$ onto the derivation algebra $\mathfrak{D}(\mathfrak{C})$ of $\mathfrak{C}$. Therefore $\mathfrak{D}(\mathfrak{C})$ is semisimple or $\{0\}$. But then $\mathbb{E}$ is semisimple by Hochschild's theorem, and its radical $\mathfrak{R}$ is $\{0\}$. That is, $\mathfrak{A}$ is semisimple.

\section{REFERENCES}

1. A. A. Albert, On right alternative algebras, Ann. of Math. (2) vol. 50 (1949) pp. 318-328.

2. R. H. Bruck and E. Kleinfeld, The structure of alternative division rings, Proceedings of the American Mathematical Society vol. 2 (1951) pp. 878-890.

3. S. Eilenberg, Extensions of general algebras, Annales de la Société Polonaise de Mathématique vol. 21 (1948) pp. 125-134.

4. Harish-Chandra, On the radical of a Lie algebra, Proceedings of the American Mathematical Society vol. 1 (1950) pp. 14-17.

5. G. Hochschild, Semisimple algebras and generalized derivations, Amer. J. Math. vol. 64 (1942) pp. 677-694.

6. N Jacobson, Completely reducible Lie algebras of linear transformations, Proceedings of the American Mathematical Society vol. 2 (1951) pp. 105-113.

7. - General representation theory of Jordan algebras, Trans. Amer. Math. Soc. vol. 70 (1951) pp. 509-530.

8. A. Malcev, On the representation of an algebra as a direct sum of the radical and a semisimple subalgebra, C. R. Acad. Sci. URSS. vol. 36 (1942) pp. 42-45.

9. R. D. Schafer, The Wedderburn principal theorem for alternative algebras, Bull. Amer. Math. Soc. vol. 55 (1949) pp. 604-614.

10. - Inner derivations of nonassociative algebras, Bull. Amer. Math. Soc. vol. 55 (1949) pp. 769-776.

11. - A theorem on the derivations of Jordan algebras, Proceedings of the American Mathematical Society vol. 2 (1951) pp. 290-294.

12. M. Zorn, Theorie der alternativen Ringe, Abh. Math. Sem. Hamburgischen Univ. vol 8 (1930) pp. 123-147.

13. - Alternative rings and related questions I: Existence of the radical, Ann of Math. (2) vol. 42 (1941) pp. 676-686.

University of Pennsylvania, Philadelphia, Pa. 